AIAA-2002-1459

\title{
Parametric Study of The Effect of Membrane Tension on Sunshield DYNAMICS
}

\author{
Brian Ross ${ }^{1}$, John D. Johnston ${ }^{1}$, James Smith. ${ }^{1}$ \\ (1) NASA - Goddard Space Flight Center, Greenbelt, MD20771 - USA
}

\begin{abstract}
The NGST sunshield is a lightweight, flexible structure consisting of pretensioned membranes supported by deployable booms. The structural dynamic behavior of the sunshield must be well understood in order to predict its influence on observatory performance. A $1 / 10^{\text {th }}$ scale model of the sunshield has been developed for ground testing to provide data to validate modeling techniques for thinfilm membrane structures. The validated models can then be used to predict the behaviour of the full scale sunshield. This paper summarizes the most recent tests performed on the $1 / 10^{\text {th }}$ scale sunshield to study the effect of membrane preload on sunshield dynamics. Topics to be covered include the test setup, procedures, and a summary of results.
\end{abstract}

\section{INTRODUCTION}

The Next Generation Space Telescope (NGST) requires a large sunshield to passively cool the telescope and detectors. A conceptual design for the NGST observatory, referred to as the 'yardstick' concept, was developed by NASA to establish a reference design for the mission and identify areas in need of technology development. The 'yardstick' concept sunshield consists of multiple layers of pretensioned, thin-film membranes that are supported by deployable booms. ' The behavior of large, thin-film membrane systems must be well understood to fully analyze and evaluate observatory performance. Dynamic models of large thin-film membrane structures are difficult to model using standard modeling techniques. One problem is that the structure exhibits nonlinear behavior due to the presence of large wrinkles produced by the tensioning forces. Modeling techniques have been developed to take into account the presence of wrinkles. ${ }^{2-4}$ However, these modeling methods have to be validated through comparison with test results. Ground testing of large lightweight structures is challenging because (1) they are not designed to withstand their own weight in a $\mathrm{l}-\mathrm{g}$ environment, (2) air has a significant effect on the structural response (e.g. damping, drag, and mass), and (3) traditional instrumentation has a significant influence on membrane behavior. Subscale systems are much less sensitive to gravity and can fit into available vacuum chambers, eliminating the negative effect of air. However other issues rise with manufacturing limitations such as film thickness that cannot be reduced. Constant thickness scaling laws ${ }^{j}$ have been developed to design subscale models by keeping a constant film thickness. These laws allow comparison of the subscale dynamics with the full size system. To mitigate risks associated with sunshield dynamics, a program of analysis and ground testing was undertaken by the government NGST team. The focus of these efforts is a subscale model of the NGST 'yardstick' concept sunshield. Several sessions of testing have been performed on this model. ${ }^{6}$ This paper describes the results of the latest series of ground tests performed to characterize the dynamic behavior of the one-tenth-scale model NGST sunshield under different membrane tension levels.

\section{TEST OBJECTIVES}

The objective of these tests was to determine the natural frequencies, structural damping, and mode shapes of the sunshield model in the frequency range of $0.10 \mathrm{~Hz}$ at three different membrane tension levels. Varying the tension level alters the dynamic response of the membranes since it modifies the stress field and hence the frequencies of the film. A secondary objective was to characterize the effect of gravity on the dynamic response of the sunshield by testing the sunshield in two different orientations (180 degrees apart) using identical test procedures

\section{TEST SETUP}

A test setup was developed to accept two orientations of the sunshield and to be used in either laboratory (in air) or T/V (thermal-vacuum) chamber environments. The setup is composed of four subsystems: (1) the test article, (2) the support structure, (3) the excitation system and (4) the instrumentation.

The test article, Figure 1, consists of a central block made of aluminum supporting four aluminum tubes in a cruciform manner. The support
$-1-$

American Institute of Aeronautics and Astronautics 
tubes are circular cross-section tubes with an outside diameter of $0.0159 \mathrm{~m}(0.625 \mathrm{in}$.$) and a wall thickness$ of $0.00165 \mathrm{~m}(0.065 \mathrm{in}$.). Four membrane layers of 13 micron thick, coated Kapton are attached to the central block, two on each side of the structure. Each tube tip has an interface system, Figure 2, composed of a ladder to keep the membrane spacing constant. Constant force springs (CFS) attached to the ladders produce a constant tensioning in the membrane layers. The baseline (CFS1) spring constant is 1.425 $\mathrm{N}(0.32 \mathrm{lb})$. These baseline springs result in average membrane stresses on the order of $70 \mathrm{kPa}$ (10 psi). The other springs that were tested are CFS2 with a spring constant of $2.85 \mathrm{~N}(0.64 \mathrm{lb})$ and CFS3 with a spring constant of $4.275 \mathrm{~N}(0.96 \mathrm{lb})$. This kite-like structure weighs approximately $4.5 \mathrm{~kg}$ (10 lbs).

The support structure, Figure 3, is a stiff framework of welded aluminum members composed of the following four sub-assemblies: base plate, column, support legs, and platform. The test stand has a footprint of $1.73 \mathrm{~m}$ by $0.76 \mathrm{~m}$ (68 in. by $30 \mathrm{in}$.) with a height of approximately $2 \mathrm{~m}$ (78 in.). The column supports the test article at a suitable location, and the support legs provide additional stiffness for the column. The excitation system (shaker) is located on the platform at the top of the column. The test article is mounted in a vertical orientation by fastening the central block directly to the shaker armature. The sunshield can be mounted on the test stand in two configurations: long side down (LSD) and short side down (SSD), Figure 4. It was designed such that its first fundamental frequency was over the frequency range of interest to avoid any coupling.

The excitation system used for these tests is a long stroke shaker capable of generating low frequency vibrations. The shaker is an ELECTROSEIS $B$ long stroke shaker manufactured by APS Dynamics (Carlsbad, CA).

The test article and stand are instrumented with a series of tri-axial accelerometers and a force transducer. The stand has accelerometers located on the base plate and along the column (Figure 5). The sunshield is instrumented with tri-axial accelerometers located at the tip of each tube and on the central block. The force transducer is located at the interface between the sunshield and the armature. A non-contact measurement system (laser vibrometer) is used to measure velocity of the outer layer membrane at different locations. The laser is operated in two different modes: (1) as a single point measurement device while the sunshield is subject to random excitation and (2) as a scanning system during sine dwell excitation at specific frequencies (mode shape recovery). The random excitation measurement locations on the outer layer membrane were marked with 20 small white targets (Figure 6).

\section{TEST PROCEDURES}

Preliminary testing was performed in the T/V chamber, in an ambient air environment, prior to installing the membranes on the sunshield. The objectives of these tests were to: (I) characterize the dynamic behavior of the test stand and to verify that its fundamental natural frequency was effectively out of the frequency range of interest for the sunshield test article and (2) to characterize the behavior of a 'tubes-only' (i.e. with membranes removed) test article. Results of the fixture survey showed the first test stand mode in $Z$ (the direction of excitation) at $10.9 \mathrm{~Hz}$ and a mode in $\mathrm{Y}$ at $9.3 \mathrm{~Hz}$. Tests were run with the 'tubes-only' test article oriented in both the long side down and short side down configurations. Results from the 'tubes-only' test showed a small effect due to gravity. The Z-bending mode of the long tube went from $3.63 \mathrm{~Hz}$ for the LSD case to 3.43 $\mathrm{Hz}$ for the SSD case. The Z-bending mode of the medium tube went from $5.75 \mathrm{~Hz}$ for the LSD case to $5.92 \mathrm{~Hz}$ for the SSD case. Additionally, a mode of the shaker armature suspension was identified at 0.4 $\mathrm{Hz}$. This mode is a rigid body mode of the sunshield moving with the armature in the $z$-direction (out-ofplane direction for the sunshield).

Once the membranes were installed on the sunshield support structure, two types of tests were run under vacuum to characterize the sunshield dynamics: (1) random excitation using the laser vibrometer to measure at fixed locations on the membrane and (2) sine dwell excitation for membrane mode shape recovery. There were four test configurations involving different combinations of CFS's and test article orientations: CFSI/LSD, CFS1/SSD, CFS2/LSD, and CFS3/LSD.

\section{RANDOM EXCITATION}

Random excitation tests were performed to measure frequency response functions from which the natural frequencies, damping coefficients, and mode shapes for the system can be identified. The tests were completed at an excitation level of $10 \mathrm{mg}$ rms in the $\mathrm{z}$-direction measured at the interface block. The following processing parameters were set and maintained for each test run:

- Processing frequency range: $0-32 \mathrm{~Hz}$

- Frequency resolution: $0.0312 \mathrm{~Hz}$

- Number of averages: 15

- Overlap: $50 \%$ 
During testing, the data acquisition system processed each channel to calculate its respective frequency response function (FRF), and coherence. The reference channel used to generate FRF's was the single force transducer located at the interface between the central block and the shaker armature (drive point).

\section{SINE DWELL EXCITATION}

Fine-scale mode shape recovery for the outer membrane layer was accomplished using the laser vibrometer in scanning mode with the test article under constant frequency sine excitation. The frequencies at which the tests were performed were determined from the random excitation test results. The laser vibrometer system has a feature called a "lock-in amplifier" that is used for the sine dwell tests. The lock-in amplifier is essentially a tracking filter that uses a reference signal to determine frequency and then determines the amplitude and phase of the response velocity relative to the reference signal. Typically the reference signal would be the force gage signal but for these tests, the excitation level was low resulting in a force gage output signal that was too low to work with the lockin amplifier. For most of these tests, the interface block (drive point) accelerometer signal was used as the reference. For the modes below $2.5 \mathrm{~Hz}$, the laser vibrometer lock-in amplifier had trouble locking in on the drive point accelerometer signal so the shaker drive source signal was used. The laser vibrometer system that was used had trouble locking on most of the lower frequencies and as a result, the 'locked-in' frequencies did not always exactly match the frequencies of the final analysis of the random data. The frequencies presented in this paper with the velocity contour figures are the frequencies that were "locked-in" on the vibrometer system. While using the lock-in amplifier, the laser vibrometer sensor head can be setup to scan across the test item using a user-defined number of points. When scanning the test item, the laser briefly dwells at each point for a user-selected time. The velocity magnitude and phase information for each point is then saved onto disk. Post processing of this data provides velocity contours that can be interpreted as mode shapes. The excitation level used for all the sine dwell tests was $15 \mathrm{mg}$ peak and was monitored using the interface block accelerometer and an oscilloscope/volt meter. Only the laser vibrometer was used for these tests, no other data was recorded. Approximately 900 points were scanned for all configurations except the CFS3/LSD, which was scanned with approximately 1400 points. The vibrometer measurement sample time was varied between .5 second and 1 second depending on the dwell frequency. Generally, for frequencies at or below $2 \mathrm{~Hz}$, a 1 second sample time was used.

\section{TEST RESULTS}

The testing that was performed resulted in a set of measured FRF's consisting of both acceleration and velocity FRF's. Before the analysis could be performed, the velocity FRF's were differentiated and combined with the accelerometer FRF's to form a complete set of acceleration FRF's. Modal parameters were then estimated for all test configurations using LMS's CADA-X modal analysis software. Tables 1-3 summarize the modes that were identified for the 3 different CFS configurations and Table 4 summarizes the modes that were identified for the CFSI/SSD configuration. The modes not marked with an asterisk are the modes that were selected in the initial analysis because they had consistent stable poles in the stability diagram. However, when the synthesized FRF's were generated from this initial list of modes, their agreement with the measured FRF's at locations on the membrane was not very good. It appeared as though there were small areas on the membrane where modes were evident at slightly shifted frequencies than those originally estimated. It was assumed that these were local modes on the membrane and so additional modes were added to the parameter table to account for these. The modes noted with an asterisk are these local modes. The inclusion of these local modes in the parameter table resulted in much better agreement between synthesized and measured FRF's at membrane locations. Also note that in Tables $1-4$, the modes marked with $t$ are the dominant modes for the system.

\section{General Comments}

Each of the configurations tested exhibited approximately 12 sunshield modes in the $0-10 \mathrm{~Hz}$ frequency range. The fundamental modes of the sunshield primarily involve the outer edges of the membranes with no participation from the support tubes. There are typically two dominant sunshield modes that involve significant interaction between the membranes and support tubes. The frequencies of these modes roughly correspond to the frequencies of the fundamental bending modes of the long and medium tubes. The remaining modes fall into two categories: membrane modes and membrane-tube interaction modes. The membrane modes generally occur in the frequency range of $0-3 \mathrm{~Hz}$ (i.e. up to the frequency of the first membrane-tube mode) and appear to be local modes often involving flapping of the membrane edges. It should be noted that there 
are a couple of membrane modes above $3 \mathrm{~Hz}$ that involve more participation from the membrane than just the edges. The membrane modes exhibit little or no participation from the support tubes. The membrane-tube interaction modes occur in the frequency range of $3-8 \mathrm{~Hz}$ and involve higher-order membrane modes that often include some participation from the long and medium length support tubes.

The test configurations of CFS1 in both LSD and SSD orientations are the same configurations that were run in previous tests of the sunshield. ${ }^{5}$ There are some differences in the results between the tests, most notably the identified low frequency modes. In the previous set of tests there was generally one mode involving both edges of either the long side or the short side of the membrane and in the recent test there are two modes, each including a different edge of either the long side or short side of membrane. This demonstrates the sensitivity of the sunshield membrane response and it's dependence on a large number of variables.

\section{Effect of Tension}

Upon examination of Tables 1-3 and Figure 7 , two main observations can be made concerning the effect of varying the membrane tension on the overall system dynamics. First, the modes involving the support tubes were not greatly affected by the change in preload. The frequency of the dominant long tubemembrane mode decreased slightly as the tension increased, which follows predictions, and the frequency of the dominant medium tube-membrane mode did not have a noticeable trend as the tension was increased. This also extends to locations on the membrane along the support tubes, such as points 5 and 16, Fig. 7. The portions of the membrane along the tubes tend to participate mainly in modes involving the tubes. The second observation that can be made is that the frequency content of the sunshield modes generally shifts up as the tension is increased and the locations where this effect is most evident are along the edge of the membrane, such as points 6 and 17. The plot for point 17 shows the largest frequency shift occurs for the second low frequency membrane mode, involving the right edge of the long side of the membrane, ranging from $1.42 \mathrm{~Hz}$ for CFS1, to $2.05 \mathrm{~Hz}$ for CFS2, to $2.56 \mathrm{~Hz}$ for CFS3. Above the frequency of the long tube, approximately $3.3 \mathrm{~Hz}$, it is difficult to distinguish trends in the frequency of the modes. This is likely due to the dominance of the support tubes in modes above this frequency and the high density of modes involving the membranes in this frequency range.
In addition to affecting the frequency of a mode, the change in tension also affects the mode shape. The frequency of the dominant long tubemembrane mode only varied $.13 \mathrm{~Hz}$ but the mode shape had more significant changes, see Figure 9. For CFS1, the mode shape of the membrane shows the center of the long side of the membrane out-ofphase with a significant area along the edges. As the tension is increased, the mode shape changes such that most of the long side of the membrane is moving together and only a very small portion of the edges is moving out-of-phase. Figure 10 shows the membrane mode shapes for the dominant medium tube-membrane mode. For the CFSl case, the shape shows the center of the short side of the membrane moving out-of-phase with a portion of the short side edges (Note that there are higher-order effects along the outer edges). As the tension is increased, the size of the center region increases until there are only small areas along both edges that are out-of-phase. Figure 11 shows both the frequency shift effect and the change in the mode shape for one of the low frequency modes of the long side of the membrane. The frequency shifts from $1.42 \mathrm{~Hz}$ to $2.56 \mathrm{~Hz}$ (from Tables 1-3) and the shape changes from just the long side, right quadrant participating to basically the entire long side with a small area along the right edge of higher magnitude.

\section{Effect of Gravity}

The effect of gravity is more complicated than the tension effects described in the previous section. Upon examination of the FRF plots, Figure 8 , it would appear that switching from the LSD configuration to the SSD configuration causes modes of the long side of the membrane to shift slightly down in frequency and modes of the short side of the membrane to shift slightly up in frequency. This trend agrees with the results of the preliminary 'tubes-only' tests. However, examination of the mode shapes reveals significantly different shapes for what would be expected to be similar modes based on the frequency. The best example of this is the first group of low frequency membrane modes. For the LSD case these modes involve the edges of the long side of the membrane but for the SSD case, these modes involve the edges of the short side of the membrane. It would appear that even though the frequencies are similar, the mode shapes of the low frequency membrane modes are greatly dependent on gravity and will have participation from whichever side of the sunshield is pointed down. The dominant membrane-support tube modes are not as affected by gravity. Figure 12 shows the membrane mode shapes for the dominant long tube-membrane mode from 
both LSD and SSD configurations. The frequencies are slightly different but the shapes are fairly similar.

\section{CONCLUSIONS}

Another testing session has been completed on the $1 / 10^{\text {th }}$ scale NGST sunshield providing further insight into the dynamics of thin-film membrane structures and additional data for analytical model validation. Regarding the test objective of characterizing the effect of membrane tension on sunshield dynamic response, the data shows that increasing the tension generally causes the modal frequencies to shift up and that the effect is not as great for the dominant membrane-support tube interaction modes, but is most noticeable in the low frequency membrane-only modes. Important insight has also been gained concerning the effect of gravity on the sunshield dynamic response. The test results show that gravity has a great effect on the dynamic response of the sunshield. This set of tests has also demonstrated the sensitivity of the dynamic response of the sunshield to a variety of parameters. Even though care was taken to follow identical procedures in assembly and setup of the test, there was a difference in results between this test and previous tests. This is valuable information for anyone performing tests on similar structures in the future.

\section{REFERENCES}

1. Bely, P.Y., Perrygo, C., and Burg, R., NGST "Yardstick" Mission, NGST Monograph- No. I, July 1999.

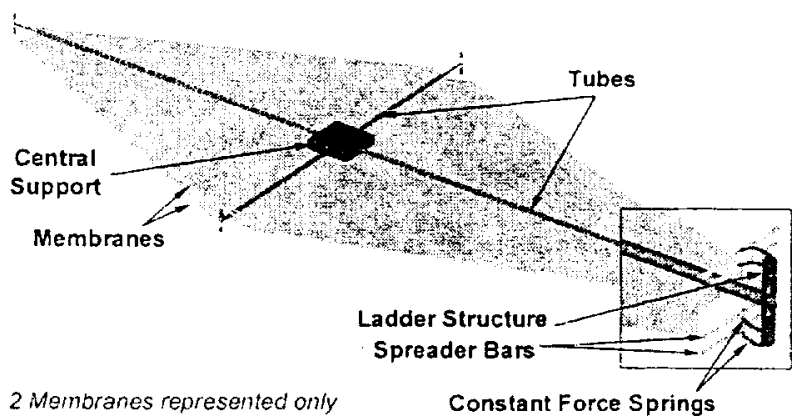

Figure 1: $1 / 10^{\text {th }}$ Scale NGST Sunshield Test Article
2. Sebastien Lienard, "Characterization of Large Thin Film Membrane Dynamic Behavior with UAI-NASTRAN Finite Element Solver," October 1999, SAE 1999-01-5518.

3. Johnston, J. and Lienard, S., "Modeling and Analysis of Structural Dynamics for a One-Tenth Scale Model NGST Sunshield", $42^{\text {ml }}$ AIAA Structures, Structural Dynamics, and Materials Conference, Seattle, WA, April 16-19, 2001, AIAA-2001-1407.

4. Adler, A.L., Mikulas, M.M., and Hedgepeth, J.M., "Static and dynamic Analysis of Partially Wrinkled Membrane Structures," $4 I^{s \prime}$ AIAA Structures, Structural Dynamics, and Materials Conference, Atlanta, GA, Paper No. AIAA2000-1810, April 2000.

5. G. Greschik, M. M. Mikulas, and R. E. Freeland, "The Nodal concept of Deployment and the Scale Model Testing of its Application to a Membrane Antenna," April 1999, AIAA 99. 1523.

6. Lienard, S., Johnston, J., Ross, B., Smith, J., "Dynamic Testing of a Subscale Sunshield for the Next Generation Space Telescope (NGST)". $42^{\text {int }}$ AIAA Structures, Structural Dynamics, and Materials Conference, Seattle, WA. Paper No. AIAA-2001-1268, April 2001.

7. LMS CADA-X Software Manual, LMS North America, 1050 Wilshire Blvd. Suite 250. Troy. MI 48084.

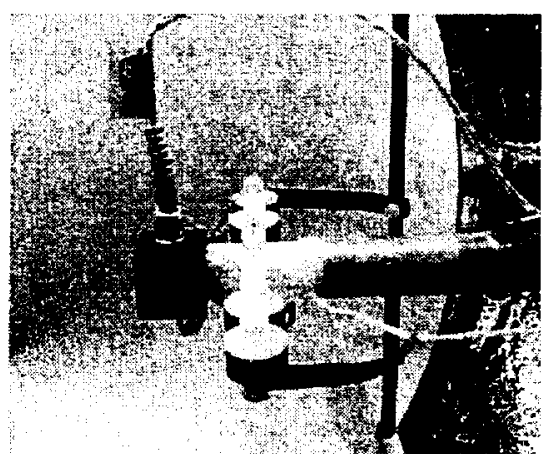

Figure 2: Tip Ladder Structure and CFS.

$-5-$

American Institute of Aeronautics and Astronautics 


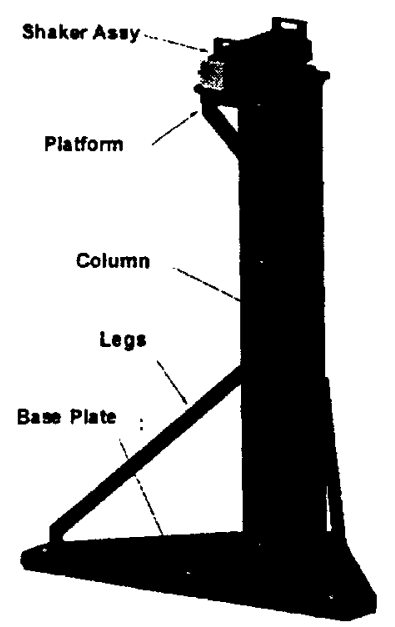

Figure 3: Subscale Sunshield Test Support Structure

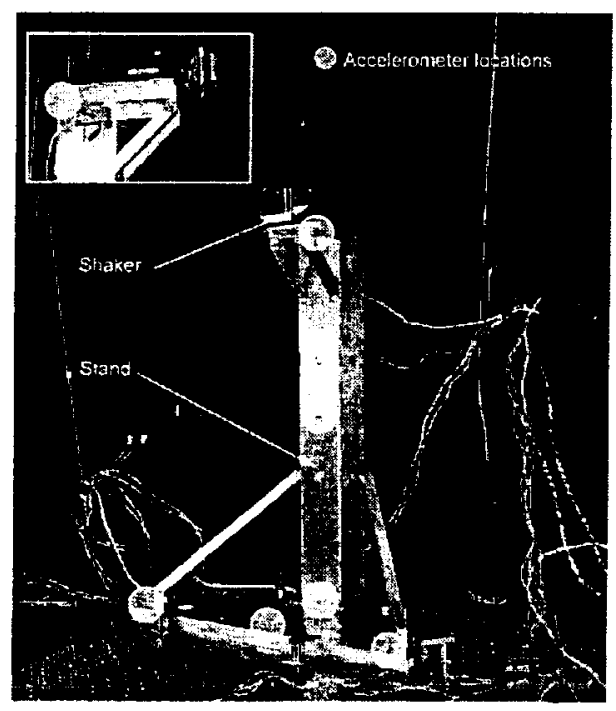

Figure 5: Support Structure Accelerometer Locations

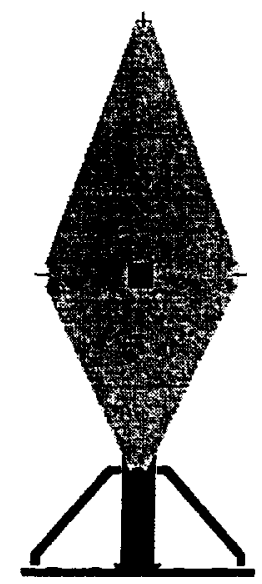

SSD

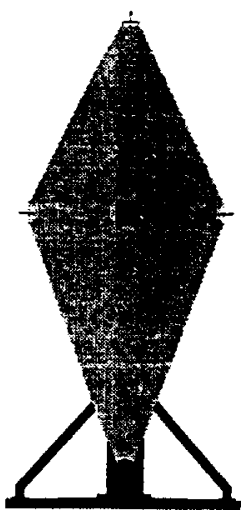

LSD
Figure 4: Sunshield Test Configurations
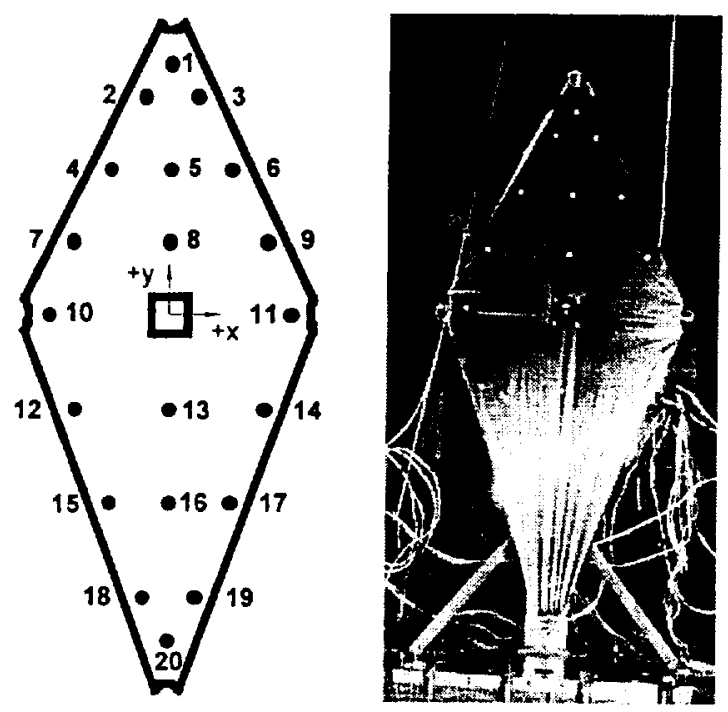

Figure 6: Membrane Target Locations for Random F.rcitation 


\begin{tabular}{|c|c|c|l|}
\hline Mode & Frequency (Hz) & Damping (\%) & \multicolumn{1}{|c|}{ Mode Shape Description } \\
\hline 1 & 1.32 & 7.29 & Flapping of membrane, long side (LS) on left edge \\
\hline 2 & 1.42 & 5.97 & Flapping of membrane, LS on right edge \\
\hline 3 & $1.51^{*}$ & 5.92 & Flapping of membrane, LS edges with right edge out of phase with left edge \\
\hline 4 & $2.25^{*}$ & 11.43 & Flapping of membrane, short side (SS) right edge in phase with LS left edge \\
\hline 5 & 2.70 & 5.75 & Flapping of membrane, SS left edge in phase with LS right edge \\
\hline 6 & $3.35^{*}$ & 3.18 & Long tube bending in Z in phase w/ LS membrane center and out of phase w/LS edges \\
\hline 7 & $3.76^{*}$ & 8.42 & Long tube bending in Z in phase w/LS membrane edges and out of phase w/LS center \\
\hline 8 & 4.15 & 4.26 & Long and medium tube bending in Y-Z direction with random membrane motion \\
\hline 9 & 4.42 & 8.17 & Membrane, SS center \\
\hline 10 & $4.64^{*}$ & 8.22 & Membrane, SS center \\
\hline 11 & $5.51^{*}$ & 2.57 & Medium tube bending in Z out of phase with SS center \\
\hline 12 & $5.74^{*}$ & 6.93 & Membrane, higher order on SS \\
\hline
\end{tabular}

Table 1. Summary of modes identified for the LSD, CFS1 configuration

\begin{tabular}{|c|c|c|l|}
\hline Mode & Frequency $(\mathbf{H z})$ & Damping (\%) & \multicolumn{1}{|c|}{ Mode Shape Description } \\
\hline 1 & $1.81^{*}$ & 10.63 & Flapping of membrane, LS on left edge \\
\hline 2 & 2.05 & 5.12 & Flapping of membrane, LS on right edge \\
\hline 3 & 2.75 & 6.10 & Flapping of membrane, short side (SS) right edge in phase with LS left edge \\
\hline 4 & $3.22^{\top}$ & 2.38 & Long tube bending in Z in phase with membrane LS \\
\hline 5 & 3.52 & 5.39 & Membrane, LS edges in phase \\
\hline 6 & 3.78 & 4.49 & Membrane, SS left edge in phase with LS edges \\
\hline 7 & $4.48^{*}$ & 2.27 & Higher order membrane with some long tube bending in Z \\
\hline 8 & $4.98^{*}$ & 6.24 & Membrane SS edges in phase with some medium tube bending in Z \\
\hline 9 & $5.40^{*}$ & 4.40 & Medium tube bending in Z in phase with membrane SS center \\
\hline 10 & $5.59^{*}$ & 3.91 & Similar to mode 8 w/ more membrane LS participation \\
\hline 11 & $6.12^{*}$ & 3.91 & Membrane SS right edge higher order \\
\hline 12 & $6.77^{*}$ & 5.30 & Membrane higher order \\
\hline 13 & 7.23 & 2.23 & Membrane SS center \\
\hline
\end{tabular}

Table 2. Summary of modes identified for LSD, CFS2 configuration

\begin{tabular}{|c|c|c|l|}
\hline Mode & Frequency $(\mathbf{H z})$ & Damping (\%) & \multicolumn{1}{|c|}{ Mode Shape Description } \\
\hline 1 & 2.01 & 13.90 & Flapping of membrane, LS on left edge \\
\hline 2 & 2.56 & 4.40 & Flapping of membrane, LS on right edge \\
\hline 3 & $3.22^{\top}$ & 2.45 & Long tube bending in Z in phase with membrane LS \\
\hline 4 & 3.62 & 5.18 & Membrane, short side (SS) right edge out of phase with LS left edge \\
\hline 5 & 4.56 & 0.30 & Long and medium tube bending in Y-Z direction in phase with membrane SS \\
\hline 6 & $4.62^{*}$ & 1.88 & Medium tube bending in $Z$ in phase with membrane SS \\
\hline 7 & 4.85 & 3.56 & Membrane SS in phase with some medium tube bending in Z \\
\hline 8 & $5.57^{\dagger}$ & 2.38 & Medium tube bending in $Z$ in phase w/ membrane SS center \& out of phase w/SS edges \\
\hline 9 & $6.16^{*}$ & 1.97 & Membrane higher order \\
\hline 10 & 6.52 & 3.16 & Membrane higher order \\
\hline 11 & $7.41^{*}$ & 3.55 & Membrane right edge higher order \\
\hline 12 & $8.10^{*}$ & 4.31 & Membrane higher order \\
\hline
\end{tabular}

Table 3. Summary of modes identifled for LSD, CFS3 configuration

\begin{tabular}{|c|c|c|l|}
\hline Mode & Frequency (Hz) & Damping (\%) & \multicolumn{1}{|c|}{ Mode Shape Description } \\
\hline 1 & 1.49 & 5.77 & Flapping of membrane, SS left edge out of phase with SS right edge \\
\hline 2 & $1.60^{*}$ & 8.96 & Flapping of membrane, SS right edge out of phase with LS right edge \\
\hline 3 & 1.89 & 6.37 & Flapping of membrane, LS right edge out of phase with LS left edge \\
\hline 4 & 2.07 & 6.29 & Flapping of membrane, LS right edge in phase with LS left edge \\
\hline 5 & 3.02 & 6.69 & Long tube bending in Z in phase w/ membrane LS edges \& out of phase w/LS center \\
\hline 6 & $3.21^{\top}$ & 3.67 & Long tube bending in Z in phase w/ membrane LS center \& out of phase w/LS edges \\
\hline 7 & $3.31^{*}$ & 7.00 & Membrane, LS center out of phase with SS edges \\
\hline 8 & 3.84 & 4.99 & Long and medium tube bending in Y-Z direction with random membrane motion \\
\hline 9 & $3.97^{*}$ & 7.60 & Long and medium tube bending in Y-Z direction with random membrane motion \\
\hline 10 & 4.79 & 5.23 & Membrane higher order on SS \\
\hline 11 & 4.85 & 5.39 & Medium tube bending in Z with membrane higher order SS \\
\hline 12 & $5.31^{*}$ & 4.72 & Membrane, SS center \\
\hline 13 & $5.85^{\prime}$ & 4.96 & Medium tube bending in Z out of phase with membrane SS center \\
\hline
\end{tabular}

Table 4. Summary of modes identified for the SSD, CFSI configuration

$-7-$

American Institute of Aeronautics and Astronautics 

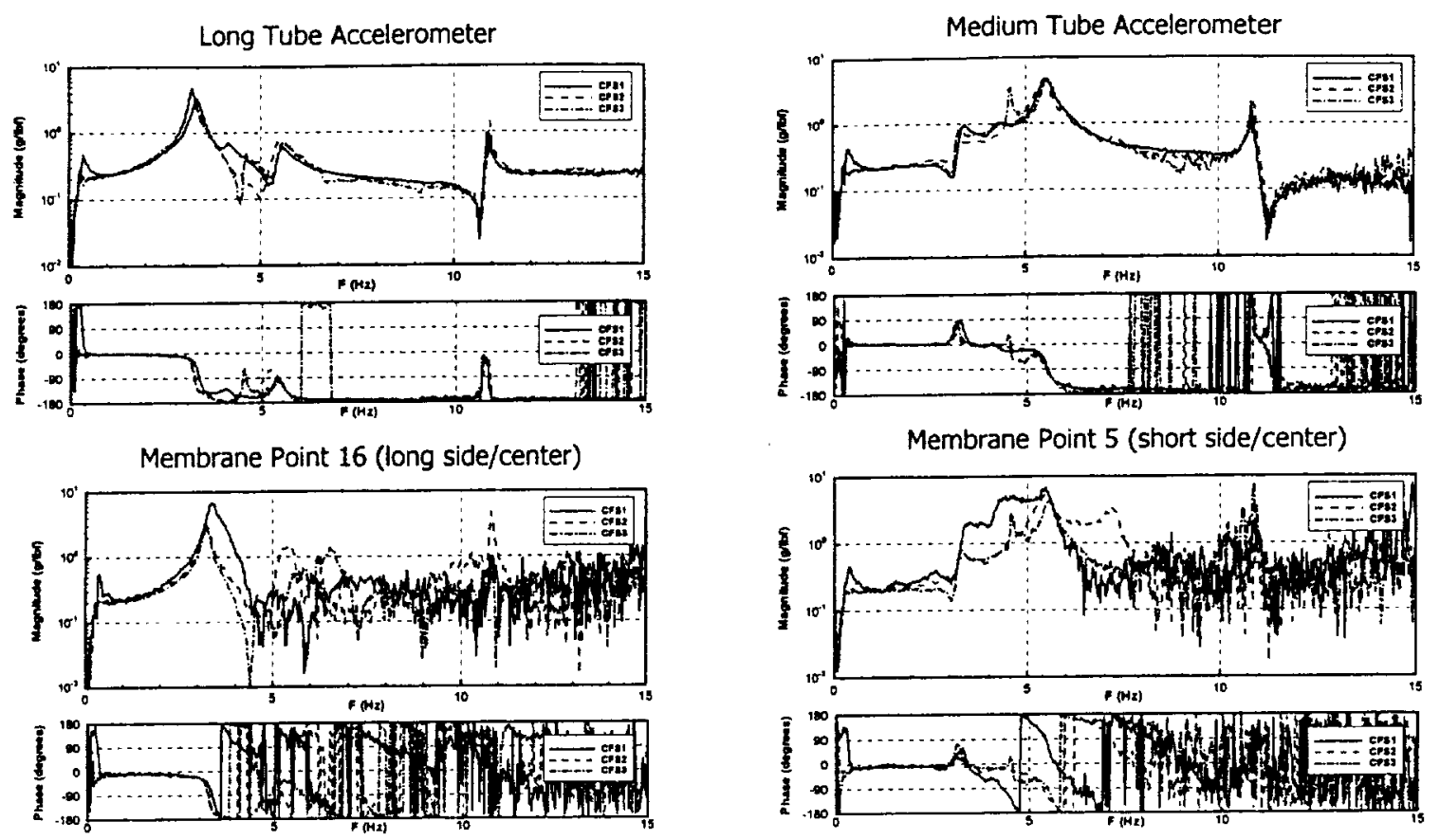

Membrane Point 17 (long side/edge)
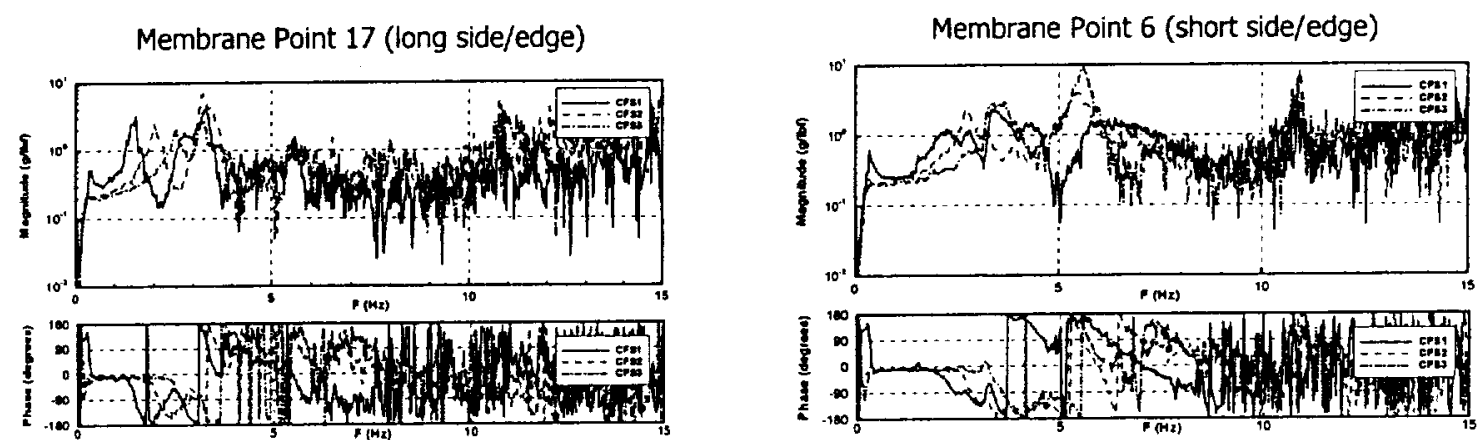

Figure 7: FRF plots summarizing tension effect 

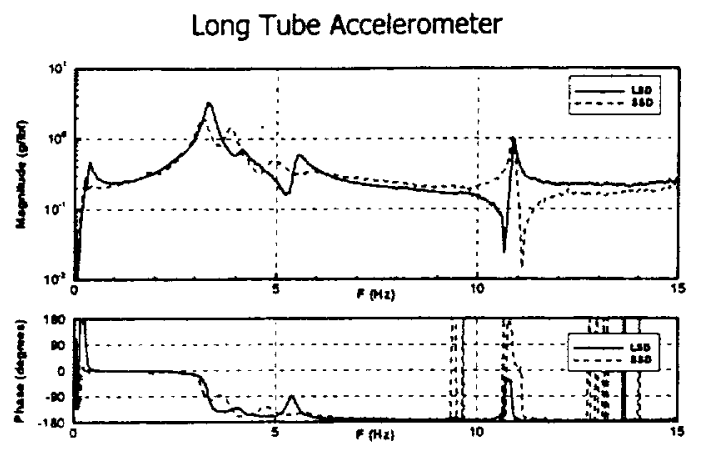

Membrane Point 16 (long side/center)
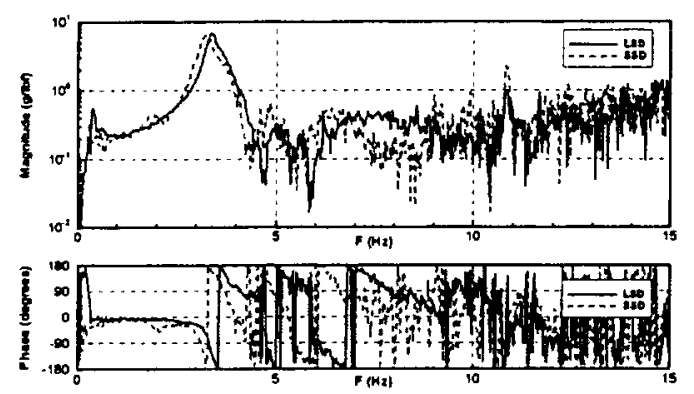

Membrane Point 17 (long side/edge)
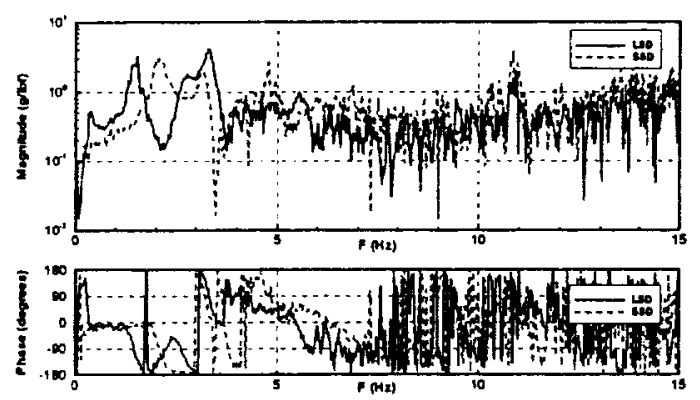

Medium Tube Accelerometer
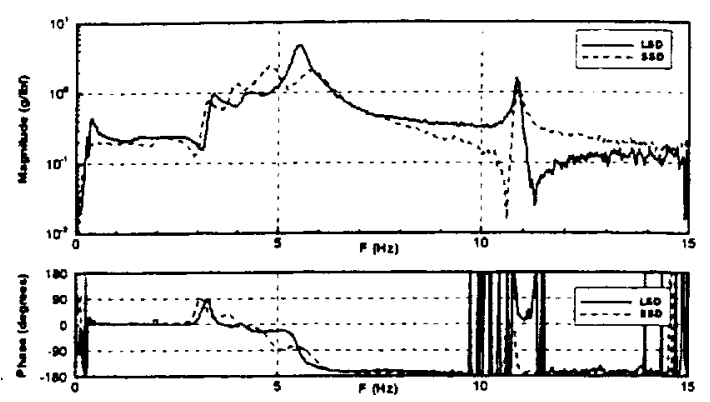

Membrane Point 5 (short side/center)
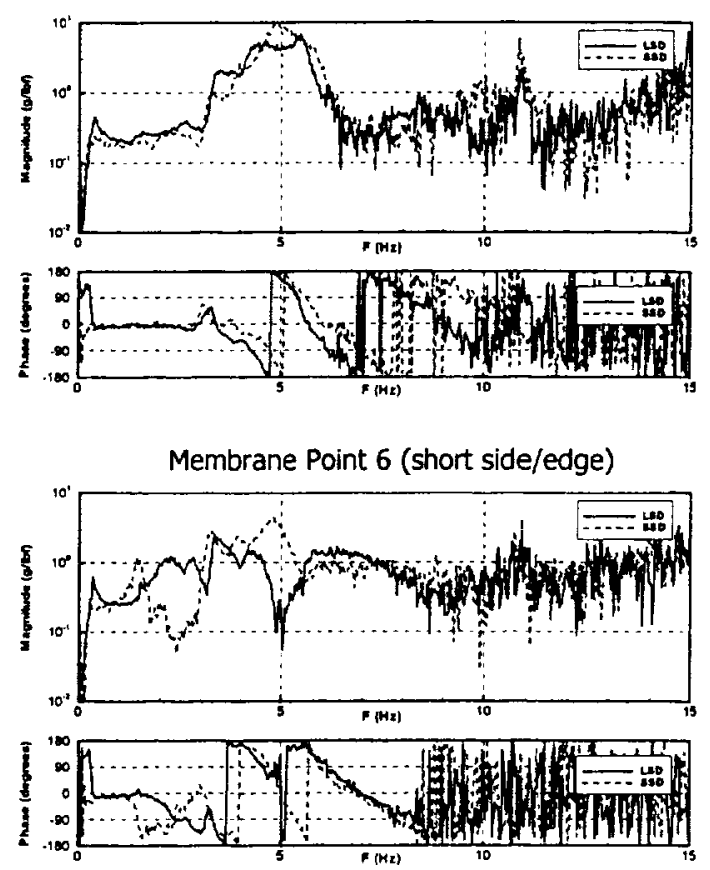

Figure 8: FRF plots summarizing the effect of gravity 

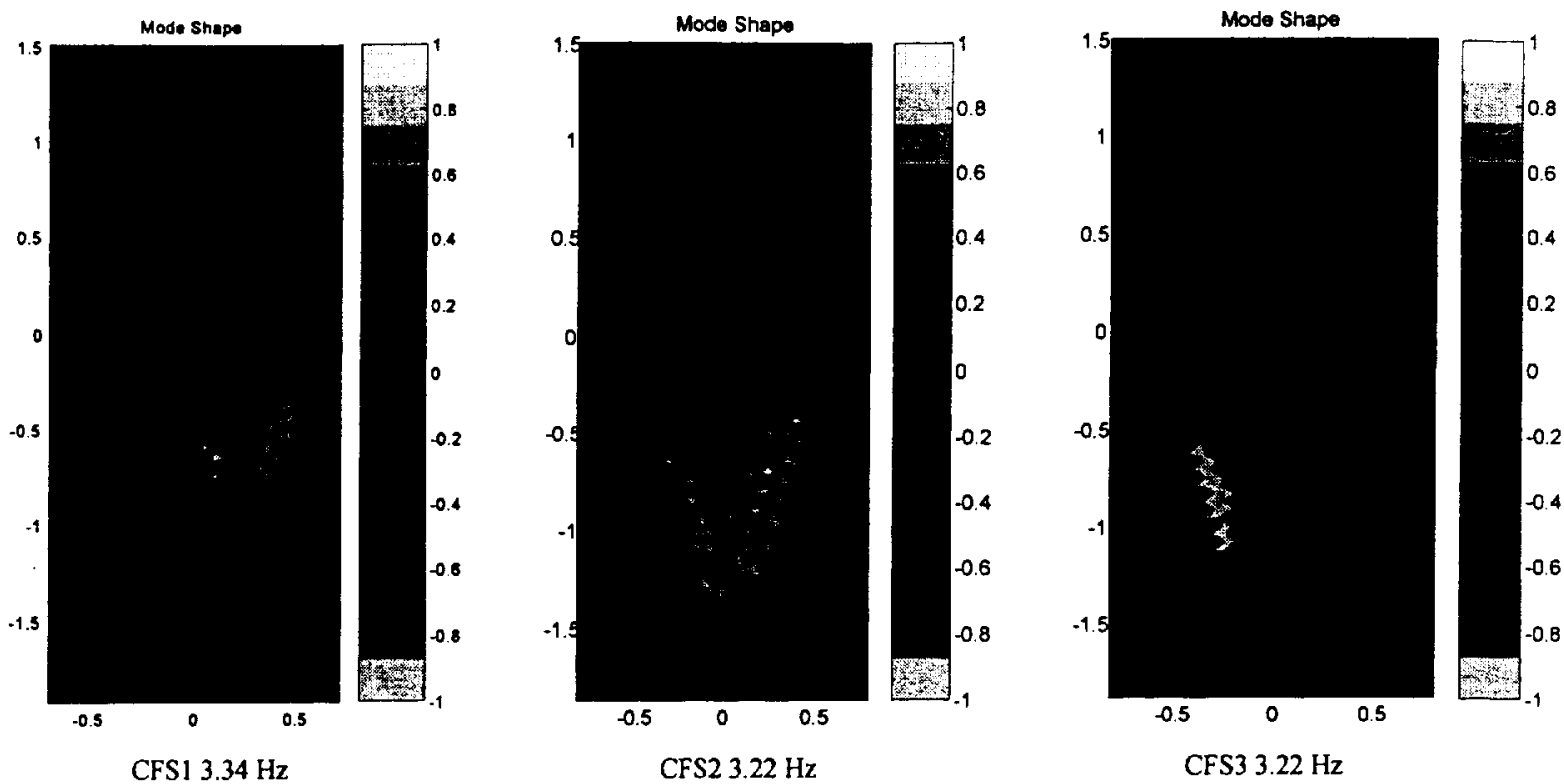

Figure 9: Tension effect comparison of membrane mode shapes for dominant long tube-membrane mode
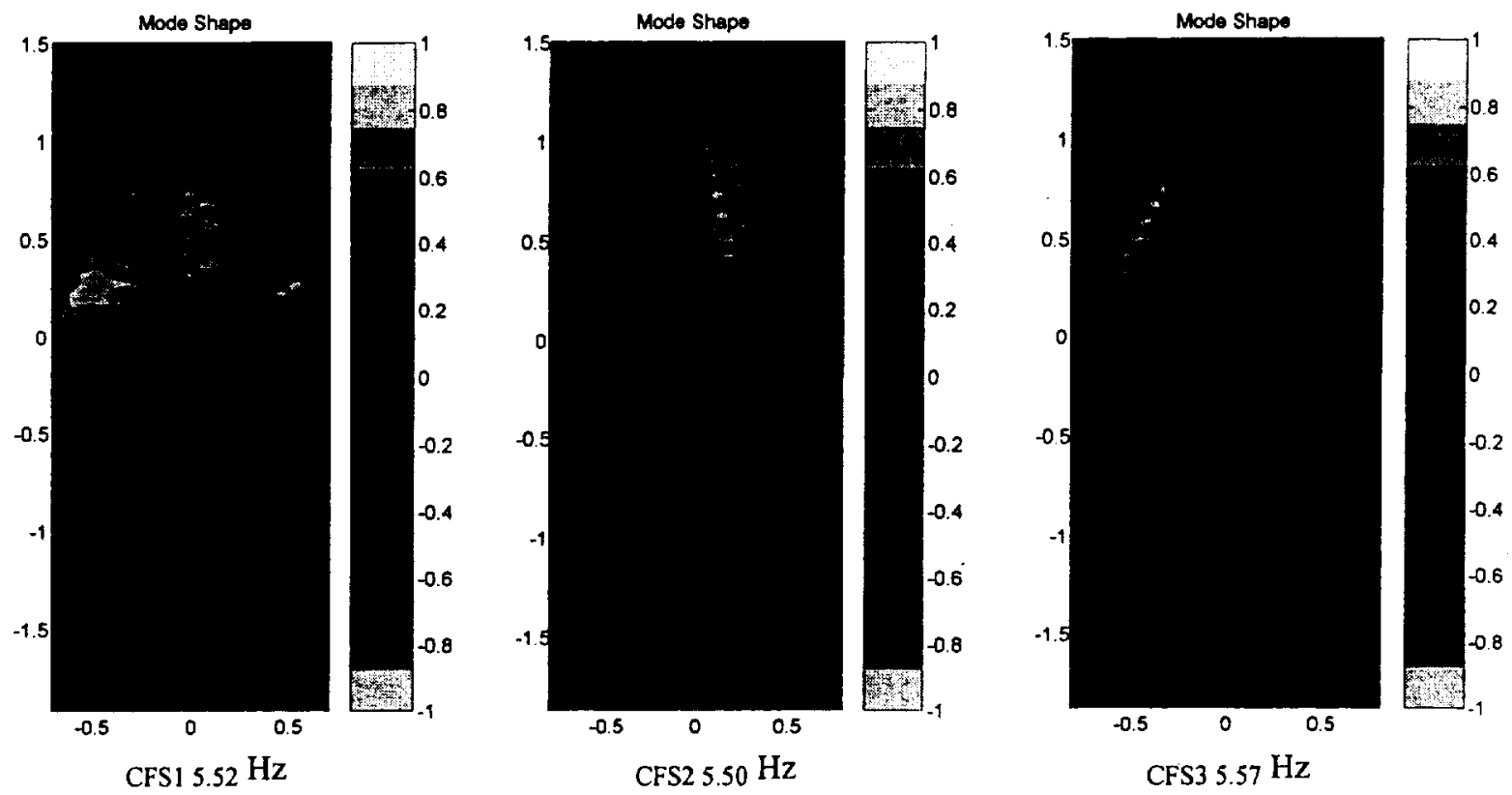

Figure 10: Tension effect comparison of membrane mode shapes for dominant medium tube-membrane mode 


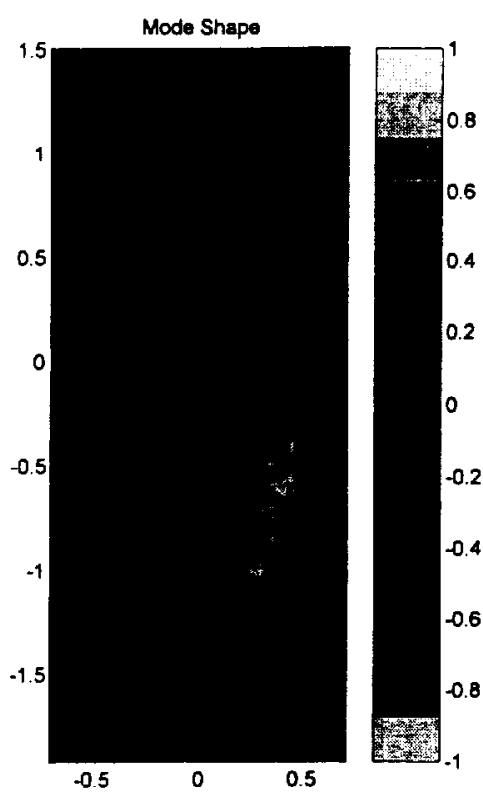

CFS $11.49 \mathrm{~Hz}$

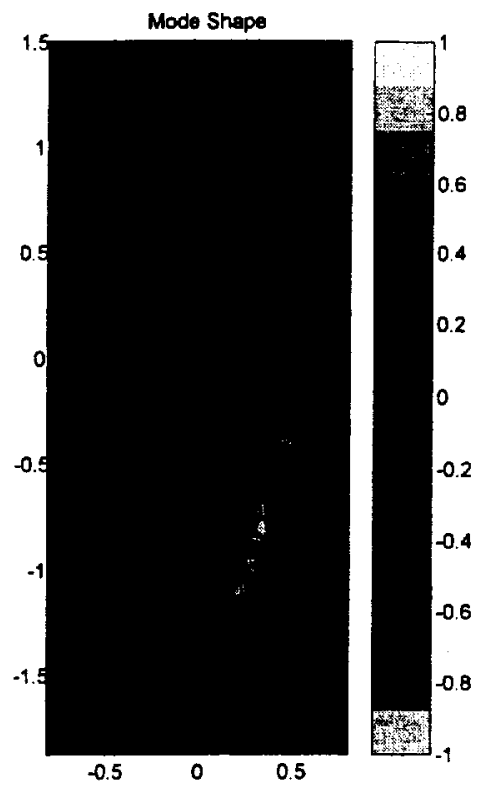

CFS2 $2.05 \mathrm{~Hz}$

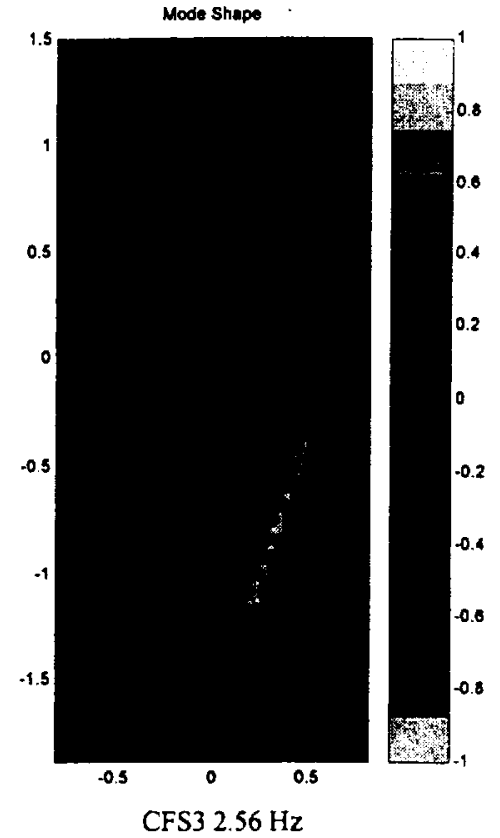

CFS $32.56 \mathrm{~Hz}$

Figure 11 : 'Tension effect comparison of membrane mode shapes for low frequency membrane mode
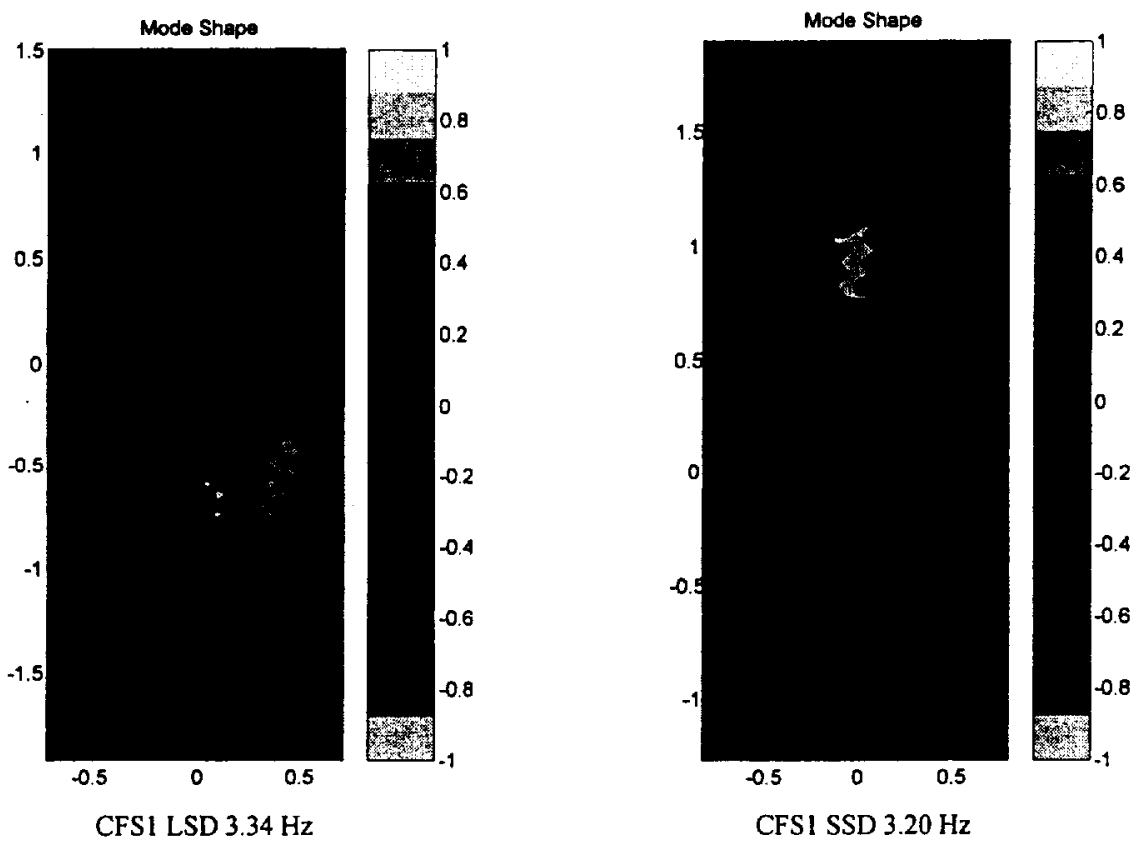

Figure 12: Gravity effect comparison of membrane mode shapes for dominant long tube-membrane mode Note: Orientations of contour plot match labeled sunshield orientation, i.e. the plots are flipped 180 degrees from each other. 